Betancourt, T. S., McBain, R., Newnham, E. A. and Brennan, R. T. (2014), Context matters: community characteristics and mental health among war-affected youth in Sierra Leone. Journal of Child Psychology and Psychiatry, 55: 217-226.

(c) 2013 The Authors. Journal of Child Psychology and Psychiatry (c) 2013 Association for Child and Adolescent Mental Health.

This is the accepted version of the following article: Betancourt, T. S., McBain, R., Newnham, E. A. and Brennan, R. T. (2014), Context matters: community characteristics and mental health among war-affected youth in Sierra Leone. Journal of Child Psychology and Psychiatry, 55: 217-226, which has been published in final form at http://dx.doi.org/10.1111/jcpp. 12131

This version was made available in the UWA Research Repository on the $26^{\text {th }}$ of May 2015 , in compliance with the publisher's policies on archiving in institutional repositories.

Use of the article is subject to copyright law. 
Word count: 6,636

Tables: 3

\section{Context matters: Community characteristics and mental health among war-affected youth in Sierra Leone}

\footnotetext{
*Theresa S. Betancourt ${ }^{1,2}$, Ryan McBain ${ }^{1,2}$, Elizabeth A. Newnham ${ }^{2,3}$, Robert T. Brennan ${ }^{2}$

${ }^{1}$ Department of Global Health and Population, Harvard School of Public Health, Boston, MA, USA

${ }^{2}$ FXB Center for Health and Human Rights, Harvard School of Public Health, Boston, MA, USA

${ }^{3}$ School of Psychology, The University of Western Australia, Perth, Australia
}

\section{*Correspondence:}

Theresa S Betancourt, ScD

Associate Professor, Department of Global Health and Population;

Director, Research Program on Children and Global Adversity, François-Xavier Bagnoud Center for Health and Human Rights. Address: Harvard School of Public Health, 651 Huntington Ave, $7^{\text {th }}$ Floor, Boston, 02115, MA, USA; Phone: +1.617.432.5003; Email: Theresa Betancourt@harvard.edu

\section{*Conflict of Interest:}

The authors declare no conflicts of interest. 


\begin{abstract}
Background. Worldwide, over one billion children and adolescents live in war-affected settings. At present, only limited research has investigated linkages between disrupted social ecology and adverse mental health outcomes among war-affected youth. In this study, we examine three community-level characteristics - social disorder and collective efficacy within the community, as reported by caregivers, and perceived stigma as reported by youth — in relation to externalizing behaviors and internalizing symptoms among male and female former child soldiers in post-conflict Sierra Leone.
\end{abstract}

Methods. 243 former child soldiers (30\% female, mean age at baseline: 16.6 years) and their primary caregivers participated in interviews in 2004 and 2008, as part of a larger prospective cohort study of war-affected youth in Sierra Leone. Two-point growth models were estimated to examine the relationship between community-level characteristics and externalizing and internalizing outcomes across the time points.

Results. Both social disorder within the community, reported by caregivers, and perceived stigma, reported by youth, positively co-varied with youths' externalizing and internalizing scores - indicating that higher levels of each at baseline and follow-up were associated with higher levels of mental health problems at both time points $(\mathrm{p}<0.05)$. The relationship between collective efficacy and mental health outcomes was non-significant $(\mathrm{p}>0.05)$.

Conclusions. This study offers a rare glimpse into the role that the post-conflict social context plays in shaping mental health among former child soldiers. Results indicate that both social disorder and perceived stigma within the community demonstrate an important relationship to externalizing and internalizing problems among adolescent ex-combatants. Moreover, these relationships persisted over a four-year period of follow up. These results underscore the 
importance of the post-conflict social environment and the need to develop post-conflict interventions that address community-level processes in addition to the needs of the individual.

Keywords: social ecology, mental health, child soldiers, post-conflict, war, externalizing, internalizing 
Increasing attention is being paid to the broader social ecology_interactions between individuals and settings of development including the family and broader community — that shapes the mental health of children exposed to war (Betancourt, Agnew-Blais, Gilman, Williams, \& Ellis, 2010; Panter-Brick, Goodman, Tol, \& Eggerman, 2011). For example, at the level of the family-unit, caregivers of war-affected youth have similarly witnessed traumatic events and may have participated in violence themselves (Betancourt \& Beardslee, 2012). The mental health consequences of these exposures can adversely impact family relationships, undermining the ability of adult members to provide emotional support for their children (Barber, 1999; Gewirtz, Forgatch, \& Wieling, 2008) and potentially leading to household violence (Panter-Brick et al., 2011). At the community level, social services and institutions such as schools, hospitals, churches and mosques are often damaged or deliberately destroyed (Barenbaum, Ruchkin, \& Schwab-Stone, 2004). Poor access to education and health care, as well as limited access to employment and income-generating opportunities, are seen as exacerbating the psychological stress created by war exposures (Collier, 1999; Stewart \& Fitzgerald, 2001). However, very little research has examined the influence of the broader social context on the mental health of war-affected youth.

One mechanism by which community ecology may influence mental health in children and adolescents relate to social disorder - the level of criminal activity, as well as general safety and conduct that pervades the community. Post-conflict communities are often characterized by crime and ongoing insecurity, and both formal and informal mechanisms that enforce normative behavior may be weak (Aron, 2003; Baker, 2007). As a result, the enduring threat to personal safety may generate significant psychological stress and foster social isolation (Cummings, Goeke-Morey, Schermerhorn, Merrilees, \& Cairn, 2009; Miller \& Rasmussen, 2010). Among 
youth who are direct victims of violence within the community, the relationship between victimization and psychological sequelae is even more direct (Betancourt et al., 2010). A second community-level channel of interest pertains to collective efficacy. Research in both Western and non-Western settings has shown that collective efficacy-informal social control and social cohesion through which communities regulate, support and supervise members - is associated with reduced rates of violent behavior and crime (Carlson, Brennan, \& Earls, 2012; Sampson, Raudenbush, \& Earls, 1997). For instance, Sampson and colleagues (1997) demonstrated that Chicago neighborhoods with higher levels of collective efficacy had lower levels of violence, even after individual-level characteristics were taken into account (Sampson et al., 1997). In Tanzania, a youth-led community health intervention aimed at increasing community efficacy resulted in positive views of youth, which were associated with increased deliberative and communicative self-efficacy among adolescent participants, as well as enhanced perceived child efficacy at the community-level (Carlson et al., 2012). Such "neighborhood effects" have been identified as influencing a variety of psychosocial outcomes among adolescents and youth, including externalizing behaviors (Kroneman, Loeber, \& Hipwell, 2004; Romano, Tremblay, Boulerice, \& Swisher, 2005) as well as internalizing symptoms (Aneshensel \& Sucoff, 1996; Sampson, Morenoff, \& Gannon-Rowley, 2002).

To date, there remains a paucity of research on the role of the community in shaping the mental health of war-affected youth. This is surprising for several reasons. First, a preponderance of post-conflict settings_-particularly those in sub-Saharan Africa, Latin America and South Asia-represent collectivist societies in which the role of community is a central feature in the culture. Second, the capacity of the community to enforce normative behavior is typically compromised in war-affected settings (Baker, 2007). Third, one might suspect that externalizing 
or internalizing may manifest as coping mechanisms following war, particularly among those responding to ongoing feelings of threat and insecurity (McCrae \& Costra, 1986).

In the present study, we investigated the role of community characteristics in shaping externalizing behaviors and internalizing symptoms in a sample of male and female former child soldiers in Sierra Leone. Specifically, we examined social disorder, collective efficacy and perceived stigma as hypothesized predictors of mental health outcomes over time, using data from 2004 to 2008. To provide an independent account of the community environment, primary caregivers of the youth were interviewed about their perception of social disorder and collective efficacy in their communities, and this secondary source of information was integrated into analyses of self-reported adolescent mental health. We hypothesized that collective efficacy would be inversely associated with externalizing and internalizing symptoms over time, while social disorder and stigma would contribute to increased risks for such problems.

\section{Method}

Sample

The present investigation was conducted as part of a prospective longitudinal study of war-affected youth in post-conflict Sierra Leone (T1: 2002, T2: 2004, T3: 2008), conducted in conjunction with the International Rescue Committee (IRC). Primary explanatory variables of interest — community characteristics—-were measured in 2004 and 2008. As such, the data were restricted to those individuals who participated in these waves ( $\mathrm{n}=243 ; 30 \%$ female; mean age 16.58 years, $\mathrm{SD}=2.62$, at $\mathrm{T} 2$ ). Participants were recruited from six districts located throughout Sierra Leone—Bo, Kenema, Kono, Bombali, Moyamba and Pujehun. This process targeted recruitment among two groups of youth: former child soldiers who received disarmament, 
demobilization and reintegration (DDR) services $(n=127)$ and self-reintegrated former child soldiers $(\mathrm{n}=116)$. The sample of DDR-served youth was obtained by pooling International Rescue Committee (IRC) registries to create a master list of all youth assisted by an interim care center from June 2001 to February 2002, the most active period of demobilization; those who were between the ages of 10 and 17 years in 2002 and who had contact information available were approached and invited to participate in the baseline assessment. At T2, the cohort of selfreintegrated youth from Makeni (Bombali district) was identified and recruited from lists obtained by a partner NGO offering outreach in the region.

Data collection comprised one-on-one interviews by a team of trained local research staff and was conducted in the local language, Krio. Participants over age 18 provided oral consent, while those under 18 provided oral assent with consent given by a primary caregiver. Research protocols and procedures received approval from the Harvard School of Public Health.

\section{Measures}

All measures described below were culturally-adapted for Sierra Leone and were forward and back-translated using a standardized protocol (Betancourt et al., 2011). Qualitative data collection, including focus groups and key informant interviews, were used to develop measures of constructs not well captured by pre-existing scales.

Youth Mental Health. Externalizing behaviors and internalizing symptoms were selfreported by youth using the Oxford Measure of Psychosocial Adjustment (OMPA), a measure developed and validated for use among former child soldiers in northern Uganda and Sierra Leone (MacMullin \& Loughry, 2004). The externalizing subscale from this measure comprises 12 items, which evaluated hostile or aggressive behavior over the past six months. The scale demonstrated good internal consistency in this sample (Chronbach's $\alpha=0.86$ in 2004, $\alpha=0.80$ 
in 2008). Examples of questions include: "Do you destroy things that belong to others?" and "Do you take things from other places without permission?" The internalizing subscale comprises 16 items, indexing youth depressive and anxiety symptoms and also showed good internal consistency ( $\alpha=0.79$ in 2004, $\alpha=0.76$ in 2008). Examples of items include: "Do you feel lonely?" and "Do you worry about too many things?"

Community Characteristics. Two aspects of community characteristics were reported by caregivers, and were measured using a variant of the Project on Human Development in Chicago Neighborhoods (PHDCN) collective efficacy subscales (R. J. Sampson et al., 1997) previously adapted for use in Tanzania (Carlson et al., 2012). The social disorder subscales ask about community criminal activity, drug and alcohol abuse, as well as general safety. In contrast, collective efficacy encompasses social cohesion and trust in the community, as well as indicators of informal social control for enforcing normative behavior (Janowitz, 1975). This construct is not merely the converse of social disorder, although the two are related: While social disorder indexes insecurity and social problems within a community; collective efficacy indexes the willingness of the community to come together to address such problems. Table 1 provides an overview of the individual items in each of these subscales.

In order to reduce self-reporting bias- e.g. that those youth who act aggressively are more likely to perceive their community and environment as hostile —we utilized reports on community characteristics provided by the primary caregivers of youth participants.

Perceived Stigma. Stigma comprised a third community-level characteristic assessed in youth self-reports via an adaptation of the Everyday Discrimination Scale (Williams, Yu, Jackson, \& Anderson, 1997), which measures differential treatment in the community toward individuals for reasons such as gender or race. In the context of this survey, the evaluated reason 
for discrimination due to involvement with an armed group. The scale has a total of nine items, for which individuals affirmed or denied "former participation in fighting forces" as the primary reason for discrimination. The measure of internal consistency for this measure was good at both time points ( $\alpha=0.89$ in $2004, \alpha=0.83$ in 2008). Questions from this scale are also included in Table 1.

Family Abuse/Family Acceptance. A measure of family abuse and neglect was adapted from the Child Trauma Questionnaire (Bernstein, Fink, Handelsman, \& Foote, 1994) and was included in the 2008 survey. There were a total of three questions on physical abuse directed toward the youth participants (alpha $=0.70)$. A measure of family acceptance was also generated based on qualitative interviews in consultation with local staff, and was implemented in both 2004 and 2008. The final measure comprised six items on perceived care, acceptance and treatment by family members $(\alpha=0.90$ in $2004, \alpha=0.86$ in 2008).

Daily Hardships. In 2008, an adaptation of the Post-War Adversities Index (Layne, Stuvland, Saltzman, Djapo, \& Pynoos, 1999) was added to assess daily hardships confronting youth. Items included hunger, housing, economic insecurity, and interpersonal adversities. The scale demonstrated good internal consistency $(\alpha=0.82)$.

War Exposures. Individual war exposures were measured using items from the Child Trauma War Questionnaire (CTWQ) (Macksoud \& Aber, 1996), first developed for use among youth in Lebanon and adapted to capture war experiences relevant to the Sierra Leonean context. Based on previous research in the present sample (Betancourt et al., 2010; Betancourt, Brennan, Rubin-Smith, Fitzmaurice, \& Gilman, 2010) three experiences comprising "toxic stressors" were selected: (i) having been a victim of rape, (ii) death of a parent due to war, and (iii) perpetration of violence (killing or injuring another person). 
Demographic Characteristics. Questions from UNICEF's Multiple Indicator Cluster Survey (MICS)(UNICEF, 2007) were utilized to identify participants' age, gender, and socioeconomic status (SES). In MICS, SES is evaluated in terms of individuals' access to food, housing, and clothing relative to others. The four items representing SES demonstrated acceptable internal consistency $(\alpha=0.74)$

\section{Statistical Analyses}

Two-point growth models were estimated to examine the relationship between explanatory variables and mental health outcomes in 2004 (baseline) and 2008 (follow-up). Rogosa and Willett (1983) demonstrated that change estimated at just two points is unbiased and that the reliability of measurement of change can be calculated. The two-point growth models used in this analysis are derived from the three-point model developed by Raudenbush, Brennan, and Barnett (Raudenbush, Brennan, \& Barnett, 1995) which used a two-level hierarchical linear model to fit a quadratic function using three time points by incorporating a measurement model based on parallel subscales accounting for unreliability of the outcomes . Subsequently, Goldberg and others (Goldberg \& Sayer, 2006; Goldberg \& Smith, 2008) have employed a similar strategy to create a linear growth model with two time points. We adopt the approach used by Betancourt and colleagues (Betancourt, Newnham, McBain, \& Brennan, in press) who used items assigned to two parallel scales matched on standard deviations and items centered on items zero. Each parallel outcome scale was divided by the number of items. Thus, the outcome is interpreted on the metric of the original items whereby a 1-unit change connotes average change from, for example, "never" to "hardly" across all items.

Estimating a slope across the two time points accommodates any secular trend in mental health symptoms across the time points as well as any changes in mental health related to 
individual predictors that do not vary over time (e.g., war exposures, demographics). Timevarying covariates (e.g., social disorder), which are observed separately at T2 and T3 are incorporated into the model and predict variation in the outcome net of the effect of both the secular trend and variation predicted by time-invariant covariates. Predictors of the slope are interpreted as either associated with a decrease in symptoms over time (T2 to T3), if negative, or an increase in symptoms, if positive.

Model building comprised three steps: In the first step, individual-level characteristics were entered including demographics and personal war experiences. In the second step, post-war factors including family abuse/neglect and daily hardships were added. Lastly, in the third step, time-varying covariates were incorporated: social disorder, collective efficacy and perceived stigma from the community, as well as family acceptance. This model-building approach facilitates examination as to whether point estimates and standard errors are stable with the inclusion of additional constructs, and whether statistical significance changes as a function of controlling for aggregated covariates.

In order to address missing data, 20 multiply imputed data sets were utilized. This approach considers the relationship of missing data to other observed characteristics in the data set, known as the assumption of data missing at random (MAR), thereby reducing bias associated with the common strategy of complete case analysis (listwise deletion), which makes the stricter assumption of data missing completely at random (MCAR). Multiple imputation accounts for sampling variability across imputations by introducing an error term for each imputed value (Rubin, 1987). All variables had missingness of less than $20 \%$, with the exception of daily hardships (30\%). Descriptive analyses were conducted using STATA 12.0/SE (StataCorp, 2011). 
Growth models were fit and multiple imputations were conducted using a limited release of HLM 7.0 software (Raudenbush, Bryk, Cheong \& Congdon, 2011)

\section{Results}

Participants were, on average, 16.6 years old in $2004(\mathrm{SD}=2.6)$, and 36\% were female. Between 2004 and 2008, externalizing behaviors depreciated by approximately 33\%, from an average OMPA externalizing score of 8.7 to 5.8 (scale range: 0-36). In contrast, internalizing symptoms depreciated by only $6 \%$, from an average score of 19.5 to 18.4 (scale range: $0-48$ ) Further descriptive characteristics of the sample are provided in Table 2.

Table 3 provides an overview of results from the final two-point growth models. In terms the externalizing outcome, the community-level measure of social disorder positively co-varied with externalizing scores, meaning that higher levels of social disorder at baseline and follow-up were associated with higher levels of externalizing behavior at both time points $(\beta=0.05$, $\mathrm{p}<0.001)$. The $\beta$-coefficient implies that living in a community that was 1 -unit higher on the social disorder scale (range: 0-6) was associated with scoring 0.05 Likert units higher on the externalizing scale at baseline (2004) and follow-up (2008). In contrast, there was no relationship between collective efficacy and externalizing $(\beta=0.01, p>0.05)$. The final community-level measure — stigma for participation in fighting forces—also positively co-varied with externalizing $(\beta=0.05, \mathrm{p}<0.001)$.

Two individual-level covariates were significantly associated with externalizing: Having been a victim of rape was predictive of lower externalizing over time $(\beta=-0.58, \mathrm{p}<0.001)$ among males, while having perpetrated violence against another was predictive of higher externalizing at baseline $(\beta=0.18, \mathrm{p}<0.001)$ among males and females with greater reduction in externalizing over time found among boys $(\beta=-0.16, p<0.01)$. At the level of the family-unit, physical abuse 
by household members and daily economic and interpersonal hardships were associated with greater externalizing behaviors at baseline $(\beta=0.04, \mathrm{p}<0.01)$ and over time, respectively $(\beta=$ $0.03, \mathrm{p}<0.01)$. With the inclusion of gender interaction terms in the final step of the modelbuilding exercise, it was further found that internalizing improved less among female victims of rape relative to male victims $(\beta=0.63, \mathrm{p}<0.05)$, whereas females who self-reported killing or injuring others improved more than their male counterparts $(\beta=-0.31, \mathrm{p}<0.05)$. Overall, the final two-point growth model accounted for $43 \%$ of variance in the intercepts (externalizing scores at baseline) and $42 \%$ of the variance in slopes (change in scores over time).

In terms of internalizing symptoms, both community-level social disorder $(\beta=0.04$, $\mathrm{p}<0.05)$ and community stigma $(\beta=0.06, \mathrm{p}<0.001)$ positively co-varied with internalizing scores over time such that higher levels of social disorder and stigma at baseline and follow-up were associated with higher levels of internalizing at both time points. The relationship between collective efficacy and internalizing was non-significant ( $>>0.05)$. At the individual-level, having been a victim of rape was associated with higher internalizing at baseline $(\beta=0.17, \mathrm{p}<0.05)$ and greater improvement over time among boys $(\beta=-0.27, \mathrm{p}<0.01)$. Additionally, individuals who had had a parent die due to war reported higher internalizing scores at baseline $(\beta=0.09$, $\mathrm{p}<0.05$ ). Lastly, those processed via Interim Care Centers (ICCs) following the war demonstrated higher internalizing scores at baseline $(\beta=0.09, \mathrm{p}<0.05)$. At the level of the family, individuals who reported physical abuse within their households had higher levels of internalizing at baseline $(\beta=0.04, p<0.05)$; those who reported higher levels of daily hardships improved less over time $(\beta=0.05, \mathrm{p}<0.05)$. Greater family acceptance at both time points inversely co-varied with internalizing $(\beta=-0.02, \mathrm{p}<0.01)$. 
Interaction terms identified several differences between males and females. First, females processed via ICCs improved less than males who received care from these locations $(\beta=0.42$, $\mathrm{p}<0.05)$. Second, females who had been a victim of rape $(\beta=0.41, \mathrm{p}<0.05)$, lost a parent as a result of the war $(\beta=0.34, \mathrm{p}<0.05)$, or reported physical abuse by family members $(\beta=0.11$, $\mathrm{p}<0.05)$ improved less over time than males who reported these same experiences. The final model for the internalizing outcome accounted for $43 \%$ of variance in the intercepts and $32 \%$ of the variance in slopes.

\section{Discussion}

This study represents one of the first analyses to examine the role of the social context in shaping war-affected adolescents' mental health trajectories. We found that, even after controlling for key risk and protective factors at the individual- and family-levels, both social disorder within the community and community-based stigma are associated with rates of youth externalizing and internalizing emotional and behavioral problems up to six years following the end of the war.

Elements of community social disorder, such as physical violence, crime, and drug and alcohol abuse have been associated with an array of adverse mental health outcomes among adolescents in Western settings (McDonald \& Richmond, 2008). However, until now it has been largely unknown whether these effects extend to non-Western contexts, or if adverse consequences are magnified following war. In the present study, we found that living in a Sierra Leonean community with higher levels of social disorder predicted both greater externalizing and internalizing among war-affected youth. Such linkages have longer term implications for health and well-being given psychophysiological research demonstrating that individuals who express elevated externalizing and internalizing also exhibit sustained levels of stress with 
detriments to longer-term physical health (Chida \& Steptoe, 2009; Player \& Peterson, 2011). Such mental health problems may also undermine interpersonal interactions (Brondolo et al., 2003) and employment prospects (Virtanen et al., 2005).

Stigma was evidenced as an important community factor shaping mental health outcomes over time. Stigma related to involvement in fighting forces has been documented in other postconflict settings such as northern Uganda and Liberia (Coulter, Persson, \& Utas, 2008). In these contexts, community programs aimed at reunification, reconciliation and forgiveness have particular promise. Furthermore, targeted youth interventions in settings like Tanzania have proved successful in enhancing youth self-perceptions and comportment within the broader community (Carlson et al., 2012). Prior intervention research in Sierra Leone has targeted and showed improvements among war-exposed children and adolescents on symptoms of posttraumatic stress disorder (Gupta \& Zimmer, 2008). However, the intervention model did not incorporate involvement of the community nor strategies for youth to improve community relations. Future interventions might incorporate the findings of this study by accounting for community dynamics and focusing on the relationship between the community context and individual-level outcomes in both intervention components and outcome measures are warranted.

In contrast to our other measures of community characteristics, no relationship was found between collective efficacy and externalizing or internalizing symptoms at baseline or follow-up. There are two main theoretical reasons for this lack of statistical significance. First, the final model concluded upon in analyses controlled for a number of other variables at the individual and household levels, and this may have weakened the relationships of interest. Second, one of the features of the present analysis is the application of caregiver reports on community characteristics. While these secondary reports are useful insofar as they represent less biased 
accounts, they also may miss insights into community interactions that youth themselves perceive, thereby increasing the likelihood of not finding a relationship.

A number of ancillary covariates also predicted baseline mental health as well as outcomes over time. At first glance, one finding that may seem particularly surprising is that receiving support from Interim Care Centers was associated with higher internalizing scores at baseline and higher externalizing scores over time. However, this result is understandable once one considers that individuals who required help from an ICC to return home at the war's end were likely less connected to their families and communities, making self-reintegration difficult. Also of interest are differences between boys and girls. For example, female victims of rape demonstrated higher internalizing and externalizing symptoms over time, relative to male victims. Evidence in western settings indicates elevated psychopathology among both male and female victims of sexual abuse (Chen et al., 2010). In the context of Sierra Leone, though, women were often repeatedly raped or kept as sex slaves during the war, and from this vantage point their experiences may have been qualitatively different from those of men (Human Rights Watch, 2003). Equally contextual may be the finding that females who killed or injured others had lower externalizing problems over time, compared to men who perpetrated these acts. Here, it may be the case that boys carried out these actions on a more routine basis and thus have greater consequences for their emotional and behavioral health.

Ultimately, heightened externalizing and internalizing problems have the potential to limit a young persons' ability to engage with educational and vocational opportunities, interpersonal relationships, and — on a larger scale—community and societal norms. As such, social disorder in the post-war community has the capacity to hamper reintegration efforts. Without a safe environment that enables adolescents to form trusting relationships, former child 
soldiers may have difficulty adjusting and thus miss opportunities to maximize the limited resources (such as social support) and opportunities (such as employment) available to them in their communities. In addition, their reaction to a hostile environment may be perceived as aggressive or uncooperative and contribute to a negative spiral of perceived stigma and distrust. Community-based programs that support safety and education around re-integration of former child soldiers have potential to bolster community cohesion and also reduce mental health risks in years following the cessation of war.

A few study limitations must be noted. First, an independent door-to-door assessment of social context would have been preferable to the reports of child soldiers and caregivers; likewise, a locally-developed survey on social context may have yielded a richer set of information for analyses. Second, although our study data collection has involved three waves of data collection, these assessments of social context were only first introduced during the second wave of data collection (2004) and then repeated in the third wave (2008). Nonetheless, this study is unique in that it includes independent reports from caregivers, in addition to the selfreported data from youth, over a four-year interval.

\section{Conclusion}

In the present study, we find that two community-level characteristics—social disorder and stigma toward former child soldiers - have a long-term impact on both externalizing and internalizing symptoms of war-affected youth. Given this enduring linkage, interventions to help war-affected youth in Sierra Leone should extend beyond the individual to increase stability and acceptance within the community. 


\section{Key Points:}

1. Research has infrequently investigated linkages between disrupted social ecology and adverse mental health outcomes among war-affected youth.

2. We examine three post-conflict, community-level characteristics-social disorder and collective efficacy within the community, as reported by caregivers, and perceived stigma as reported by youth - in relation to externalizing and internalizing symptoms among male and female former child soldiers in post-conflict Sierra Leone.

3. Both social disorder within the community and perceived stigma directed at youth by the community were related to higher externalizing and internalizing scores at baseline (2004) and four-year follow-up (2008).

4. These results demonstrate the importance of contextualizing mental health interventions within the broader community in which war-affected young persons are situated. Interventions targeted solely towards individual youth, without engagement or consideration of the role of the community, are liable to have a limited impact.

\section{Acknowledgments}

We extend our gratitude to our participants, as well as the local research assistants who conducted interviews in Sierra Leone, and our project coordinators and colleagues at the International Rescue Committee. This study was funded by the United States Institute of Peace, USAID/DCOF, Grant \#1K01MH077246-01A2 from the National Institute of Mental Health, the International Rescue Committee, the François-Xavier Bagnoud Center for Health and Human Rights and an Early Career Fellowship from the National Health and Medical Research Council Australia. 


\section{References}

Aneshensel, C.S., \& Sucoff, C.A. (1996). The neighborhood context of adolescent mental health. Journal of Health and Social Behavior, 37, 293-310.

Aron, J. (2003). Building institutions in post-conflict African economies. Journal of International Development, 15, 471-485.

Baker, B. (2007). Conflict and African police culture: the cases of Uganda, Rwanda and Sierra Leone. In M. O'Neil \& M. Marks (Eds.), Police Occupational Culture: New Debates and Directions. Oxford: Elsevier Science.

Barber, Brian K. (1999). Political violence, family relations, and Palestinian child functioning. Journal of Adolescent Research, 14, 206-230.

Barenbaum, J., Ruchkin, V., \& Schwab-Stone, M. (2004). The psychological aspects of children exposed to war: Practice and policy initiatives. Journal of Child Psychology \& Psychiatry, 45, 41-62.

Bernstein, D.P., Fink, L., Handelsman, L, \& Foote, J. (1994). Initial reliability and validity of a new retrospective measure of child abuse and neglect. American Journal of Psychiatry, $151,1132-1136$.

Betancourt, T.S., Borisova, II, Williams, T.P., Brennan, R.T., Whitfield, T.H., de la Soudiere, M., . . Gilman, S.E. (2010). Sierra Leone's former child soldiers: a follow-up study of psychosocial adjustment and community reintegration. Child Development, 81, 10771095.

Betancourt, T.S., Agnew-Blais, J., Gilman, S. E., Williams, D.R., \& Ellis, B.H. (2010). Past horrors, present struggles: the role of stigma in the association between war experiences 
and psychosocial adjustment among former child soldiers in Sierra Leone. Social Science \& Medicine, 70, 17-26.

Betancourt, T.S., \& Beardslee, W.R. (2012). The consequences of concentrated adversity on child and adolescent mental health. In V. Maholmes \& R. King (Eds.), The Oxford Handbook of Poverty and Child Development. New York: Oxford University Press.

Betancourt, T.S., Brennan, R.T., Rubin-Smith, J., Fitzmaurice, G.M., \& Gilman, S.E. (2010). Sierra Leone's former child soldiers: a longitudinal study of risk, protective factors, and mental health. Journal of the American Academy of Child and Adolescent Psychiatry., 49, 606-615.

Brondolo, E., Rieppi, R., Erickson, S.A., Bagiella, E., Shapiro, P.A., McKinley, P., \& Sloan, R.P. (2003). Hostility, interpersonal interactions, and ambulatory blood pressure. Psychosomatic Medicine, 65, 1003-1011.

Carlson, M., Brennan, R. T., \& Earls, F. (2012). Enhancing adolescent self-efficacy and collective efficacy through public engagement around HIV/AIDS competence: a multilevel, cluster randomized-controlled trial. Social Science \& Medicine, 75, 10781087.

Chen, L.P., Murad, M.H., Paras, M.L., Colbenson, K.M., Sattler, A.L., Goranson, E.N., . . . Zirakzadeh, A. (2010). Sexual abuse and lifetime diagnosis of psychiatric disorders: systematic review and meta-analysis. Mayo Clinic Proceedings, 85, 618-629.

Chida, Y., \& Steptoe, A. (2009). The association of anger and hostility with future coronary heart disease: a meta-analytic review of prospective evidence. Journal of the American College of Cardiology, 53, 936-946. 
Collier, P. (1999). On the economic consequences of civil war. Oxford Economic Papers, 51, 168-183.

Coulter, C., Persson, M., \& Utas, M. (2008). Young female fighters in African wars. Uppsala, Sweden: The Nordic Africa Institute.

Cummings, E. Mark, Goeke-Morey, Marcie C., Schermerhorn, Alice C., Merrilees, Christine E., \& Cairn, Ed. (2009). Children and political violence from a social ecological perspective: implications from research on children and families in Northern Ireland. Clinical Child and Family Psychology Review, 12, 16-38.

Gewirtz, A., Forgatch, M., \& Wieling, E. (2008). Parenting practices as potential mechanisms for child adjustment following mass trauma. Journal of Marital and Family Therapy, 34(2), $177-192$.

Goldberg, A E , \& Sayer, A. (2006). Lesbian couples' relationship quality across the transition to parenthood Journal of Marriage and Family, 68, 87-100.

Goldberg, A E, \& Smith, J Z. (2008). The social context of lesbian mothers' anxiety during early parenthood. Parenting - Science and Practice, 8, 213-239.

Gupta, L., \& Zimmer, C. (2008). Psychosocial intervention for war-affected children in Sierra Leone. British Journal of Psychiatry, 192(3), 212-216.

Human Rights Watch. (2003). We'll Kill You If You Cry. from http://www.hrw.org/reports/2003/01/15/well-kill-you-if-you-cry

Janowitz, M. (1975). Sociological theory and social control. American Journal of Sociology, 81, $82-108$. 
Kroneman, L., Loeber, R., \& Hipwell, A.E. (2004). Is neighborhood context differently related to externalizing problems and delinquency for girls compared with boys? Clinical Child and Family Psychology Review, 7, 109-122.

Layne, C.M., Stuvland, R., Saltzman, W., Djapo, N., \& Pynoos, R. S. (1999). Adolescent Post War Adversities Scale: Unpublished instrument.

Macksoud, M.S., \& Aber, J.L. (1996). The war experiences and psychosocial development of children in Lebanon. Child Development, 67(1), 70-88.

MacMullin, C., \& Loughry, M. (2004). Investigating psychosocial adjustment of former child soldiers in Sierra Leone and Uganda. Journal of Refugee Studies, 17(4), 460-472.

McCrae, R.R., \& Costra, P.T. (1986). Personality, coping, and coping effectiveness in an adult sample. Journal of Personality, 54(2), 385-404.

McDonald, C. C., \& Richmond, T. R. (2008). The relationship between community violence exposure and mental health symptoms in urban adolescents. Journal of Psychiatric and Mental Health Nursing, 15, 833-849.

Miller, Kenneth E., \& Rasmussen, Andrew. (2010). War exposure, daily stressors, and mental health in conflict and post-conflict settings: Bridging the divide between trauma-focused and psychosocial frameworks. Social Science \& Medicine, 70, 7-16.

Panter-Brick, C., Goodman, A., Tol, W. A., \& Eggerman, M. (2011). Mental health and childhood adversities: a longitudinal study of school children in Kabul, Afghanistan. Journal of the American Academy of Child \& Adolescent Psychiatry, 50, 349-363.

Player, M. S., \& Peterson, L. E. (2011). Anxiety disorders, hypertension, and cardiovascular risk: a review. International Journal of Psychiatry \& Medicine, 41, 365-377. 
Raudenbush, S.W., Brennan, R.T., \& Barnett, R.C. (1995). A multivariate hierarchical model for studying psychological change within married couples. Journal of Family Psychology, 9, $161-174$.

Raudenbush, S.W., Bryk, A.S., Cheong, Y.F., \& Congdon, R.T. (2011). HLM: Hierarchical Linear and Nonlinear Modeling. Chicago, IL: Scientific Software International.

Romano, E., Tremblay, R.E., Boulerice, B., \& Swisher, R. (2005). Multilevel correlates of childhood physical aggression and prosocial behavior. Journal of Abnormal Child Psychology, 33, 565-578.

Rubin, D.B. (1987). Multiple Imputation for Nonresponse in Surveys. New York: Wiley.

Sampson, R. J., Raudenbush, S. W., \& Earls, F. (1997). Neighborhoods and violent crime: a multilevel study of collective efficacy. Science, 277(5328), 918-924.

Sampson, R.J., Morenoff, J.D., \& Gannon-Rowley, T. (2002). Assessing "neighborhood effects": social processes and new directions in research. Annual Review of Sociology, 28, 443478.

StataCorp. (2011). Stata Statistical Software: Release 12.0. College Station, Texas: StataCorp LP.

Stewart, F., \& Fitzgerald, V. (Eds.). (2001). The Economic and Social Consequences of Conflict. Oxford: Oxford University Press.

T.S., Betancourt, S.E., Meyers-Ohki, A., Stevenson, C., Ingabire, F., Kanyanganzi, M., Munyana, ... W.R., Beardslee. (2011). Using mixed-methods research to adapt and evaluate a family strengthening intervention in Rwanda. African Journal of Traumatic Stress, 2, 32-45. 
UNICEF. (2007). Sierra Leone: Multiple Indicator Cluster Survey 2005. New York: United Nations Children's Fund.

Virtanen, M., Kivimaki, M., Elovainio, M., Vahtera, J., Kokko, K., \& Pulkkinen, L. (2005). Mental health and hostility as predictors of temporary employment: evidence from two prospective studies. Social Science \& Medicine, 61(10), 2084-2095.

Williams, D.R., Yu, Y., Jackson, J.S., \& Anderson, N.B. (1997). Racial differences in physical \& mental health: Socioeconomic status, stress, and discrimination. Journal of Health Psychology, 2(3), 335-351. 
Table 1. Items on each of the Community Characteristics subscales

\section{Social Disorder}

1. How much of a problem are some groups of young people gathered around in this community?

2. In the past month were thefts or other crimes in your community?

3. Is drunkenness a problem in your community?

4. During the past month, how often was there a heated argument between your neighbors?

5. In the past six months were there rapes in your community?

6. How safe are you and your house from crime in the streets?

\section{Collective efficacy}

1. Is this community a good place to raise a child?

2. Whenever something in this town gets spoiled, do the elders join together to repair it?

3. Do you believe (trust) people in this community?

4. If there were a fight in front of your house do your neighbors normally come to separate a fight?

\section{Stigma}

1. How often are you treated with smaller respect than other people?

2. How often are you treated in a way that is not as fine as other people?

3. How often do you receive bad face when you go to the market or other places where they sell?

4. How often do people act as if you lack sense?

5. How often do people act as if they are afraid of you?

6. How often do people act as if you are dishonest?

7. How often do people pretend to be better than you?

8. How often do people say things to you that you do not like?

9. How often do people threaten you? 
Table 2. Characteristics of the sample and distributions of main variables at each assessment reported as mean (SD) or frequency $(\%)$

\begin{tabular}{lccc}
\hline Demographics & Range of Scale & $\mathbf{2 0 0 4}$ & $\mathbf{2 0 0 8}$ \\
\hline Age & --- & $16.6(2.6)$ & $20.9(3.2)$ \\
Female & -- & $73(30.0 \%)$ & $73(30.0 \%)$ \\
\hline Community Characteristics & & $\mathbf{2 0 0 4}$ & $\mathbf{2 0 0 8}$ \\
\hline Social disorder & 0 (low) to 6 (high) & $2.2(1.4)$ & $2.1(1.4)$ \\
Collective efficacy & 0 (low) to 4 (high) & $3.7(0.6)$ & $3.8(0.6)$ \\
Perceived Stigma & 0 (low) to 9 (high) & $2.4(2.8)$ & $1.0(2.0)$ \\
\hline Outcomes of Interest & & $\mathbf{2 0 0 4}$ & $\mathbf{2 0 0 8}$ \\
\hline Externalizing Symptoms & 0 (low) to 36 (high) & $8.7(6.0)$ & $5.8(4.2)$ \\
Internalizing Symptoms & 0 (low) to 48 (high) & $19.5(7.3)$ & $18.4(6.2)$ \\
\hline
\end{tabular}


Table 3a.Two-point growth model of risk and protective factors associated with externalizing behaviors among war-affected youth.

\begin{tabular}{|c|c|c|c|c|c|c|}
\hline & Model 1 & & Model 2 & & Model 3 & \\
\hline Demographics & Intercept (SE) & Slope (SE) & Intercept (SE) & Slope (SE) & Intercept (SE) & Slope (SE) \\
\hline Intercept & $0.002(0.018)$ & $-0.242(0.034) * * *$ & $0.001(0.018)$ & $-0.241(0.034)^{* * *}$ & $0.002(0.016)$ & $-0.242(0.037)^{* * *}$ \\
\hline Age & $-0.010(0.005)+$ & $0.013(0.012)$ & $-0.005(0.006)$ & $0.018(0.011)$ & $-0.006(0.005)$ & $0.014(0.009)$ \\
\hline Female & $0.045(0.051)$ & $-0.051(0.093)$ & $0.071(0.055)$ & $-0.025(0.092)$ & $0.045(0.047)$ & $0.333(0.128)^{*}$ \\
\hline SES & $0.008(0.008)$ & $-0.021(0.015)$ & $0.004(0.008)$ & $-0.023(0.015)$ & $0.001(0.007)$ & $-0.022(0.013)+$ \\
\hline ICC & $0.070(0.043)$ & $0.158(0.078)^{*}$ & $0.071(0.041)+$ & $0.162(0.078)^{*}$ & $0.091(0.037)^{*}$ & $0.125(0.075)+$ \\
\hline \multicolumn{7}{|l|}{ War Exposures } \\
\hline Victim of Rape & $0.110(0.062)+$ & $-0.325(0.121)^{*}$ & $0.091(0.066)$ & $-0.329(0.125)^{* *}$ & $0.082(0.059)$ & $-0.584(0.140)^{* * *}$ \\
\hline Killed/Injured Others & $0.284(0.041)^{* * *}$ & $-0.221(0.078)^{* *}$ & $0.263(0.040)^{* * *}$ & $-0.232(0.076)^{* *}$ & $0.178(0.038)^{* * *}$ & $-0.159(0.069)^{*}$ \\
\hline Female*Victim of Rape & & & & & & $0.674(0.210)^{* *}$ \\
\hline Female*Killed/Injured Others & & & & & & $-0.296(0.128)^{*}$ \\
\hline \multicolumn{7}{|l|}{ Post-War Factors } \\
\hline Family Abuse & & & $0.043(0.017)^{*}$ & $0.040(0.027)$ & $0.040(0.015)^{* *}$ & $0.037(0.021)+$ \\
\hline Hardship & & & $0.014(0.007)^{*}$ & $0.005(0.019)$ & $0.009(0.006)$ & $0.028(0.010)^{* *}$ \\
\hline \multicolumn{7}{|l|}{ Time-Varying Covariates } \\
\hline Social Disorder & & & & & \multicolumn{2}{|c|}{$0.049(0.012)^{* * *}$} \\
\hline Collective Efficacy & & & & & \multicolumn{2}{|c|}{$0.007(0.029)$} \\
\hline Family Acceptance & & & & & \multicolumn{2}{|c|}{$-0.010(0.007)$} \\
\hline Child Soldier Stigma & & & & & \multicolumn{2}{|c|}{$0.052(0.009)^{* * *}$} \\
\hline Prop. of Variance Explained & 0.22 & 0.15 & 0.30 & 0.18 & 0.43 & 0.42 \\
\hline
\end{tabular}


Table 3b.Two-point growth model of risk and protective factors associated with internalizing symptoms among war-affected youth.

\begin{tabular}{|c|c|c|c|c|c|c|}
\hline & Model 1 & & Model 2 & \multicolumn{3}{|c|}{ Model 3} \\
\hline Demographics & Intercept (SE) & Slope (SE) & Intercept (SE) & Slope (SE) & Intercept (SE) & Slope (SE) \\
\hline Intercept & $0.000(0.020)$ & $-0.061(0.032)+$ & $0.000(0.019)$ & $-0.062(0.031)^{*}$ & $0.001(0.019)$ & $0.001(0.037)$ \\
\hline Age & $-0.002(0.006)$ & $-0.015(0.010)$ & $0.004(0.006)$ & $-0.011(0.010)$ & $-0.009(0.006)$ & $-0.011(0.013)$ \\
\hline Female & $0.054(0.062)$ & $-0.156(0.094)$ & $0.084(0.063)$ & $-0.124(0.092)$ & $0.023(0.052)$ & $0.164(0.101)$ \\
\hline SES & $0.007(0.009)$ & $0.011(0.014)$ & $0.004(0.011)$ & $0.005(0.014)$ & $0.005(0.010)$ & $-0.004(0.017)$ \\
\hline ICC & $0.053(0.047)$ & $0.007(0.078)$ & $0.051(0.044)$ & $0.008(0.075)$ & $0.093(0.040)^{*}$ & $0.002(0.070)$ \\
\hline Female*ICC & & & & & & $0.423(0.162)^{*}$ \\
\hline \multicolumn{7}{|l|}{ War Exposures } \\
\hline Victim of Rape & $0.186(0.072)^{*}$ & $-0.174(0.103)+$ & $0.160(0.073)^{*}$ & $-0.193(0.098)+$ & $0.174(0.069)^{*}$ & $-0.271(0.103)^{* *}$ \\
\hline Killed/Injured Others & $0.163(0.044)^{* * *}$ & $-0.076(0.071)$ & $0.144(0.041)^{* * *}$ & $-0.084(0.070)$ & $0.035(0.044)$ & $-0.024(0.080)$ \\
\hline Death of Parent & $0.080(0.047)+$ & $0.042(0.079)$ & $0.080(0.055)$ & $0.013(0.078)$ & $0.094(0.045)^{*}$ & $-0.027(0.076)$ \\
\hline Female*Death of Parent & & & & & $-0.181(0.097)+$ & $0.336(0.147)^{*}$ \\
\hline Female*Victim of Rape & & & & & & $0.406(0.164)^{*}$ \\
\hline \multicolumn{7}{|l|}{ Post-War Factors } \\
\hline Family Abuse & & & $0.049(0.016)^{* *}$ & $0.036(0.025)$ & $0.044(0.015)^{* *}$ & $0.018(0.025)$ \\
\hline Hardship & & & $0.023(0.009)^{*}$ & $0.024(0.022)$ & $0.011(0.008)$ & $0.052(0.013)^{* * *}$ \\
\hline Female*Family Abuse & & & & & & $0.105(0.046)^{*}$ \\
\hline \multicolumn{7}{|l|}{ Time-Varying Covariates } \\
\hline Social Disorder & & & & & \multicolumn{2}{|c|}{$0.037(0.015)^{*}$} \\
\hline Collective Efficacy & & & & & \multicolumn{2}{|c|}{$0.061(0.035)+$} \\
\hline Family Acceptance & & & & & \multicolumn{2}{|c|}{$-0.020(0.007)^{* *}$} \\
\hline Child Soldier Stigma & & & & & \multicolumn{2}{|c|}{$0.057(0.009)^{* * *}$} \\
\hline Prop. of Variance Explained & 0.16 & 0.01 & 0.31 & 0.12 & 0.43 & 0.32 \\
\hline
\end{tabular}

$+p<0.10, * p<0.05, * * p<0.01, * * * p<0.001$. SE stands for robust standard error. Coefficient of \pm 1 represents a one-unit increase/decrease on each individual item of the internalizing outcome on the Oxford Psychosocial Adjustment Scale. 